\title{
Research Article Splitting of Traffic Flows to Control Congestion in Special Events
}

\author{
Ciro D'Apice, Rosanna Manzo, and Luigi Rarità \\ Dipartimento di Ingegneria Elettronica e Ingegneria Informatica, Università degli Studi di Salerno, \\ Via Ponte Don Melillo, 84084 Fisciano, Italy \\ Correspondence should be addressed to Rosanna Manzo, rmanzo@unisa.it
}

Received 24 December 2010; Accepted 12 February 2011

Academic Editor: Marianna Shubov

Copyright (C 2011 Ciro D'Apice et al. This is an open access article distributed under the Creative Commons Attribution License, which permits unrestricted use, distribution, and reproduction in any medium, provided the original work is properly cited.

\begin{abstract}
We deal with the optimization of traffic flows distribution at road junctions with an incoming road and two outgoing ones, in order to manage special events which determine congestion phenomena. Using a fluid-dynamic model for the description of the car densities evolution, the attention is focused on a decentralized approach. Two cost functionals, measuring the kinetic energy and the average travelling times, weighted with the number of cars moving on roads, are considered. The first one is maximized with respect to the distribution coefficient, and the second is minimized with respect to the same control parameter. The obtained results have been tested by simulations of urban networks. Decongestion effects are also confirmed estimating the time a car needs to cross a fixed route on the network.
\end{abstract}

\section{Introduction}

The vehicles congestion is one of the most important problem of modern cities, challenging many researchers to find techniques to control it. A solution to the problem is represented by the use of more lanes and the construction of crossings, but in many areas the solution is not feasible, and moreover the building and expanding of roads to accommodate the increase of vehicles is more expensive. In particular, the presence of unexpected heavy traffic in situations such as accidents leads to delay in the arrival of the emergency services and supplies to where they are needed. In the case of special events, escorts, closures of roads, traffic directions, and control functions can be performed, when necessary, to ensure the safe and efficient movement of vehicles, splitting the traffic flows at intersections in such way to improve the viability. An example is in Figure 1, where policemen are involved to manage traffic at junctions. 


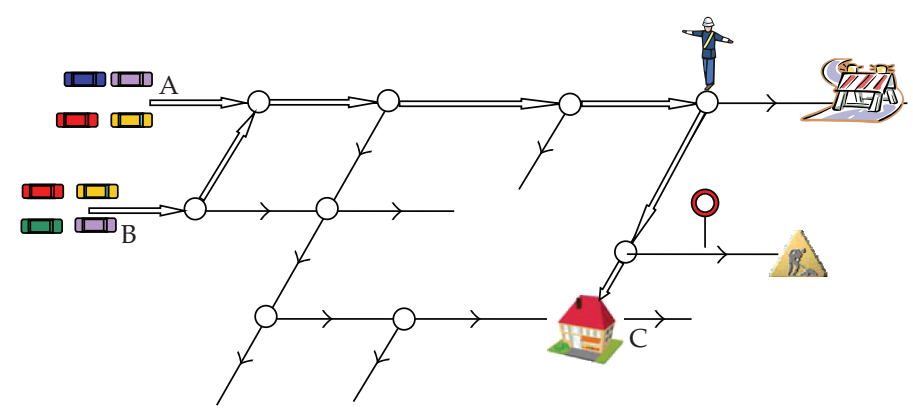

Figure 1: Example of car flows redistribution.

In this context, using a fluid dynamic model able to foresee the traffic density evolution on road networks (see [1-4]), we propose a strategy to redistribute in an optimal way flows at junctions. According to the adopted model, the car densities on each road follow a conservation law (see [5]), while dynamics at junctions is uniquely solved using the following rules:

(A) the incoming traffic at a node is distributed to outgoing roads according to some distribution coefficients;

(B) drivers behave so as to maximize the flux through the junction.

If a junction $J$ is of $1 \times 2$ type (namely, one incoming road, 1 , and two outgoing ones, 2 and 3), rule (A) is expressed by a distribution parameter $\alpha$, indicating the percentage of cars going from road 1 to road 2 . Assigning initial densities for incoming and outgoing roads and using rule (B), we finally compute the asymptotic solution as function of $\alpha$.

Here, considering the distribution coefficient as control parameter, we aim to redirect traffic at junctions of $1 \times 2$ type in order to improve urban traffic and face emergency situations. In particular, we analyze two optimization problems over a fixed time horizon: minimizing an objective function $W_{1}$, estimating the kinetic energy; maximizing a functional $W_{2}$, measuring the average travelling time of drivers, weighted with the number of cars moving on roads. Indeed, we prove that both functionals are optimized for the same value of $\alpha$.

Some control strategies for the right of way parameters and distribution coefficients have already been treated in $[6,7]$, where three cost functionals, related to average velocity, average travelling time, and flux, have been introduced for $1 \times 2$ and $2 \times 1$ junctions. Cost functionals $W_{1}$ and $W_{2}$ have been studied in [8] for the optimal control of green and red phases of traffic lights, while in [9] parameters of $2 \times 2$ junctions have been optimized for the fast transit of emergency vehicles along an assigned path in case of car accidents.

The analysis of the functionals $W_{1}$ and $W_{2}$ on a whole network is a very hard task, so we follow a decentralized approach: an exact solution is found for single $1 \times 2$ junctions and asymptotic $W_{1}$ and $W_{2}$. The global (sub)optimal solution for networks is obtained by localization: the exact optimal solution is applied locally for each time at each junction of $1 \times 2$ type.

The analytical optimization results are then tested by simulations (for numerics, see [10-12]), analyzing optimal and random distribution coefficients. The first ones are given by the optimization algorithm; the second ones consider, at the beginning of the simulation process, random values of $\alpha$, kept constant during the simulation. Then effects of the decentralized approach on the global performance of two networks have been analyzed. 
Simulation results for a symmetric topology show that, assuming random distribution coefficients, the congestion of one road can determine high traffic densities on the whole network, while decongestion phenomena occur when optimal $\alpha$ values are used. In the case study of a portion of the Salerno urban network in Italy, characterized by an asymmetric topology, with $1 \times 2$ and $2 \times 1$ junctions, some interesting aspects arise: random coefficients frequently provoke hard congestions, as expected; optimal distribution coefficients allow a local redistribution of traffic flows. While random simulation curves of the cost functional $W_{1}$ are always lower than the optimal one, the optimal curve of $W_{2}$ is higher than some random ones. This is not surprising because, at $2 \times 1$ junctions, traffic densities can remain high. Hence, for such a network, (locally) optimal solutions alleviate critical traffic situations, but the aim of the global optimization of $W_{1}$ and $W_{2}$ is not achieved. Moreover, using an algorithm (see [13]) for tracing car trajectories on a network, some simulations are run to test how the total travelling time of a driver is influenced by distribution coefficients. As intuition suggests, the time for covering a path of a single driver decreases when optimal $\alpha$ values are used.

The paper is organized as follows. Section 2 is devoted to the description of the model for road networks and to the construction of solutions to Riemann Problems $1 \times 2$ junctions. In Section 3, we define the cost functionals $W_{1}$ and $W_{2}$ and optimize them with respect to the distribution coefficients at a single junction. Simulation results for complex networks are presented in Section 4. Section 5 ends the paper through conclusions.

\section{A Riemann Solver for Road Networks}

A road network is described by a couple $(\supset, \partial)$, where $\supset$ represents the set of roads, modelled by intervals $\left[a_{i}, b_{i}\right] \subset \mathbb{R}, i=1, \ldots, N$, and $\partial$ is the collection of junctions.

Indicating by $\rho=\rho(t, x) \in\left[0, \rho_{\max }\right]$ the density of cars, $\rho_{\max }$ the maximal density, $f(\rho)=\rho v(\rho)$ the flux with $v(\rho)$ the average velocity, the traffic dynamics is described on each road by the conservation law (Lighthill-Whitham-Richards model, [3, 4]):

$$
\partial_{t} \rho+\partial_{x} f(\rho)=0
$$

We assume that: (F) $f$ is a strictly concave $C^{2}$ function such that $f(0)=f\left(\rho_{\max }\right)=0$.

Choosing $\rho_{\max }=1$ and $v(\rho)=1-\rho$, a flux function ensuring $(\mathrm{F})$ is

$$
f(\rho)=\rho(1-\rho), \quad \rho \in[0,1],
$$

which has a unique maximum $\sigma=1 / 2$.

In order to capture the dynamics at a junction, we solve Riemann Problems (RPs), Cauchy Problems with a constant initial datum for each incoming and outgoing road, the basic ingredient for the solution of Cauchy Problems by Wave-Front-Tracking algorithms.

Consider a junction $J$ of $n \times m$ type, that is, with $n$ incoming roads $I_{\varphi}, \varphi=1, \ldots, n, m$ outgoing roads, $I_{\psi}, \psi=n+1, \ldots, n+m$, and initial datum $\rho_{0}=\left(\rho_{1,0}, \ldots, \rho_{n, 0}, \rho_{n+1,0}, \ldots, \rho_{n+m, 0}\right)$.

Definition 2.1. A Riemann Solver (RS) for the junction $J$ is a map RS : $[0,1]^{n} \times[0,1]^{m} \rightarrow$ $[0,1]^{n} \times[0,1]^{m}$ that associates to Riemann data $\rho_{0}=\left(\rho_{1,0}, \ldots, \rho_{n, 0}, \rho_{n+1,0}, \ldots, \rho_{n+m, 0}\right)$ at $J$ a vector $\hat{\rho}=\left(\widehat{\rho}_{1}, \ldots, \hat{\rho}_{n, 0}, \hat{\rho}_{n+1}, \ldots, \hat{\rho}_{n+m}\right)$ so that the solution on an incoming road $I_{\varphi}, \varphi=1, \ldots, n$, is the wave $\left(\rho_{\varphi, 0}, \hat{\rho}_{\varphi}\right)$ and on an outgoing one $I_{\psi}, \psi=n+1, \ldots, n+m$ is the wave $\left(\hat{\rho}_{\psi}, \rho_{\psi, 0}\right)$. 
We require the following conditions hold true: $(C 1) \operatorname{RS}\left(\operatorname{RS}\left(\rho_{0}\right)\right)=\operatorname{RS}\left(\rho_{0}\right)$; $(C 2)$ on each incoming road $I_{\varphi}, \varphi=1, \ldots, n$, the wave $\left(\rho_{\varphi, 0}, \hat{\rho}_{\varphi}\right)$ has negative speed, while on each outgoing road $I_{\psi}, \psi=n+1, \ldots, n+m$, has the wave $\left(\hat{\rho}_{\psi}, \rho_{\psi, 0}\right)$ has positive speed.

If $m \geq n$, a possible RS at $J$ is defined by the following rules (see [1]):

(A) traffic is distributed at $J$ according to some coefficients, collected in a traffic distribution matrix $A=\left(\alpha_{j, i}\right), i=1, \ldots, n, j=n+1, \ldots, n+m, 0<\alpha_{j, i}<1$, $\sum_{j=n+1}^{n+m} \alpha_{j, i}=1$. The $i$ th column of $A$ indicates the percentages of traffic that, from the incoming road $I_{i}$, distribute to the outgoing roads;

(B) fulfilling (A), drivers maximize the flux through $J$.

Focus on a $1 \times 2$ junction $J$. We indicate the cars density on the incoming road 1 by $\rho_{1}(t, x) \in[0,1],(t, x) \in \mathbb{R}^{+} \times I_{1}$, and on the outgoing roads $\psi, \psi=2,3$, by $\rho_{\psi}(t, x) \in[0,1]$, $(t, x) \in \mathbb{R}^{+} \times I_{\psi}$.

Consider the flux function (2.2) and let $\left(\rho_{1,0}, \rho_{2,0}, \rho_{3,0}\right)$ be the initial densities at $J$. The maximal flux values on roads are defined by

$$
\begin{gathered}
\gamma_{1}^{\max }= \begin{cases}f\left(\rho_{1,0}\right) & \text { if } 0 \leq \rho_{1,0} \leq \frac{1}{2}, \\
f\left(\frac{1}{2}\right) & \text { if } \frac{1}{2} \leq \rho_{1,0} \leq 1,\end{cases} \\
\gamma_{\psi}^{\max }= \begin{cases}f\left(\frac{1}{2}\right) & \text { if } 0 \leq \rho_{\psi, 0} \leq \frac{1}{2}, \psi=2,3, \\
f\left(\rho_{\psi, 0}\right) & \text { if } \frac{1}{2} \leq \rho_{\psi, 0} \leq 1, \psi=2,3 .\end{cases}
\end{gathered}
$$

If $\alpha \in] 0,1$ [ and $1-\alpha$ indicate, respectively, the percentage of cars that, from road 1 , goes to the outgoing roads 2 and 3 , the fluxes solution to the RP at $J$ are

$$
\widehat{\gamma}=\left(\widehat{\gamma}_{1}, \alpha \widehat{\gamma}_{1},(1-\alpha) \widehat{\gamma}_{1}\right)
$$

where

$$
\widehat{\gamma}_{1}=\min \left\{r_{1}^{\max }, \frac{r_{2}^{\max }}{\alpha}, \frac{r_{3}^{\max }}{1-\alpha}\right\}
$$

Hence, $\widehat{\rho}=f^{-1}(\widehat{\gamma})$ is found as follows (see $\left.[1,2]\right)$ :

$$
\begin{gathered}
\widehat{\rho}_{1} \in\left\{\begin{array}{lc}
\left.\left.\left\{\rho_{1,0}\right\} \cup\right] \tau\left(\rho_{1,0}\right), 1\right] & \text { if } 0 \leq \rho_{1,0} \leq \frac{1}{2}, \\
{\left[\frac{1}{2}, 1\right]} & \text { if } \frac{1}{2} \leq \rho_{1,0} \leq 1,
\end{array}\right. \\
\widehat{\rho}_{\psi} \in \begin{cases}{\left[0, \frac{1}{2}\right]} & \text { if } 0 \leq \rho_{\psi, 0} \leq \frac{1}{2}, \psi=2,3, \\
\left\{\rho_{\psi, 0}\right\} \cup\left[0, \tau\left(\rho_{\psi, 0}\right)[\right. & \text { if } \frac{1}{2} \leq \rho_{\psi, 0} \leq 1, \psi=2,3,\end{cases}
\end{gathered}
$$


where $\tau:[0,1] \rightarrow[0,1]$ is the map such that $f(\tau(\rho))=f(\rho)$ for every $\rho \in[0,1]$ and $\tau(\rho) \neq \rho$ for every $\rho \in[0,1] \backslash\{1 / 2\}$.

Finally, on the incoming road 1 , the solution is given by the wave $\left(\rho_{1,0}, \widehat{\rho}_{1}\right)$, while on the outgoing road $\psi, \psi=2,3$, the solution is represented by the wave $\left(\widehat{\rho}_{\psi}, \rho_{\psi, 0}\right)$.

\section{Distribution Parameters Optimization}

Fix a $1 \times 2$ junction $J$ and an initial datum $\left(\rho_{1,0}, \rho_{2,0}, \rho_{3,0}\right)$. We define the cost functional $W_{1}(t)$ and $W_{2}(t)$, which measure, respectively, the kinetic energy and the average travelling time weighted with the number of cars moving on roads:

$$
\begin{gathered}
W_{1}(\mathrm{t})=\sum_{k=1}^{3} \int_{I_{k}} f\left(\rho_{k}(t, x)\right) v\left(\rho_{k}(t, x)\right) d x, \\
W_{2}(t)=\sum_{k=1}^{3} \int_{I_{k}} \frac{\rho_{k}(t, x)}{v\left(\rho_{k}(t, x)\right)} d x .
\end{gathered}
$$

For a fixed time horizon $[0, T]$, with $T$ sufficiently big, consider the traffic distribution coefficient $\alpha$ as control. We aim to maximize $W_{1}(T)$ and to minimize $W_{2}(T)$ separately. The functionals assume the form:

$$
\begin{gathered}
W_{1}(T)=\sum_{i=1}^{3} f\left(\widehat{\rho}_{i}\right) v\left(\widehat{\rho}_{i}\right)=\frac{1}{2} \sum_{i=1}^{3} \widehat{\gamma}_{i}\left(1-s_{i} \sqrt{1-4 \widehat{\gamma}_{i}}\right) \\
W_{2}(T)=\sum_{i=1}^{3} \frac{\widehat{\rho}_{i}}{v\left(\widehat{\rho}_{i}\right)}=\sum_{i=1}^{3} \frac{1+s_{i} \sqrt{1-4 \widehat{\gamma}_{i}}}{1-s_{i} \sqrt{1-4 \widehat{\gamma}_{i}}}
\end{gathered}
$$

where $s_{1}$ and $s_{\psi}, \psi=2,3$, are given by

$$
\begin{aligned}
& s_{1}=\left\{\begin{aligned}
+1 \quad & \text { if } \rho_{1,0} \geq \frac{1}{2} \\
& \text { or } \rho_{1,0}<\frac{1}{2} \text { and } r_{1}^{\max }>\min \left\{\frac{r_{2}^{\max }}{\alpha}, \frac{r_{3}^{\max }}{1-\alpha}\right\}, \\
-1 \quad & \text { if } \rho_{1,0}<\frac{1}{2} \text { and } \gamma_{1}^{\max } \leq \min \left\{\frac{r_{2}^{\max }}{\alpha}, \frac{r_{3}^{\max }}{1-\alpha}\right\},
\end{aligned}\right. \\
& s_{\psi}= \begin{cases}+1 \quad & \text { if } \rho_{\psi, 0}>\frac{1}{2} \text { and } \frac{\gamma_{\psi}^{\max }}{\alpha_{\psi}} \leq \min \left\{\gamma_{1}^{\max }, \frac{\gamma_{\psi^{\prime}}^{\max }}{\alpha_{\psi^{\prime}}}\right\}, \quad \psi^{\prime} \neq \psi, \\
-1 \quad & \text { if } \rho_{\psi, 0} \leq \frac{1}{2}, \\
& \text { or } \rho_{\psi, 0}>\frac{1}{2} \text { and } \frac{\gamma_{\psi}^{\max }}{\alpha_{\psi}}>\min \left\{\gamma_{1}^{\max }, \frac{\gamma_{\psi^{\prime}}^{\max }}{\alpha_{\psi^{\prime}}}\right\}, \quad \psi^{\prime} \neq \psi,\end{cases}
\end{aligned}
$$


with

$$
\alpha_{\psi}= \begin{cases}\alpha & \text { if } \psi=2 \\ 1-\alpha & \text { if } \psi=3\end{cases}
$$

According to the solution of the RP at $J$, we have

$$
\begin{gathered}
W_{1}(T)=\frac{\widehat{\gamma}_{1}}{2}\left(1-s_{1} \sqrt{1-4 \widehat{\gamma}_{1}}\right)+\frac{\alpha \widehat{\gamma}_{1}}{2}\left(1-s_{2} \sqrt{1-4 \alpha \widehat{\gamma}_{1}}\right)+\frac{(1-\alpha) \widehat{\gamma}_{1}}{2}\left(1-s_{3} \sqrt{1-4(1-\alpha) \widehat{\gamma}_{1}}\right), \\
W_{2}(T)=\frac{1+s_{1} \sqrt{1-4 \widehat{\gamma}_{1}}}{1-s_{1} \sqrt{1-4 \widehat{\gamma}_{1}}}+\frac{1+s_{2} \sqrt{1-4 \alpha \widehat{\gamma}_{1}}}{1-s_{2} \sqrt{1-4 \alpha \widehat{\gamma}_{1}}}+\frac{1+s_{3} \sqrt{1-4(1-\alpha) \widehat{\gamma}_{1}}}{1-s_{3} \sqrt{1-4(1-\alpha) \widehat{\gamma}_{1}}},
\end{gathered}
$$

where $\widehat{\gamma}_{1}$ is given by (2.5). The values of $\alpha$, which optimize $W_{1}(T)$ and $W_{2}(T)$, are reported in the following theorem (for the sketch of the proof, see the appendix).

Theorem 3.1. Fix a $1 \times 2$ junction J. Assuming $T$ sufficiently big, the cost functionals $W_{1}(T)\left(W_{2}(T)\right)$ is maximized (minimized) for $\alpha=1 / 2$, with the exception of the following cases (for some of them, the optimal control does not exist but it is approximated):

(a) if $\gamma_{3}^{\max } \leq \gamma_{1}^{\max } / 2<\gamma_{1}^{\max } \leq \gamma_{2}^{\max }, \alpha=\alpha_{1}+\varepsilon$;

(b) if $\gamma_{2}^{\max }<\gamma_{1}^{\max } / 2<\gamma_{1}^{\max } \leq \gamma_{3}^{\max }, \alpha=\alpha_{2}$;

(c) if $\gamma_{2}^{\max }<r_{3}^{\max }<\gamma_{1}^{\max }$, we distinguish three subcases:

(c1) if $\gamma_{1}^{\max }-\gamma_{3}^{\max } \geq \gamma_{2}^{\max }, \alpha=\alpha_{3}$;

(c2) if $\gamma_{1}^{\max }-\gamma_{3}^{\max }<\gamma_{2}^{\max }=\gamma_{1}^{\max } / 2, \alpha=(1 / 2)-\varepsilon$;

(c3) if $\gamma_{1}^{\max }-r_{3}^{\max }<\gamma_{2}^{\max } \leq \gamma_{1}^{\max } / 2, \alpha=\alpha_{2}-\varepsilon$;

(d) if $\gamma_{3}^{\max }<\gamma_{2}^{\max }<\gamma_{1}^{\max }$, we distinguish two subcases:

(d1) if $\gamma_{1}^{\max }-\gamma_{3}^{\max } \geq \gamma_{2}^{\max }, \alpha=\alpha_{3}+\varepsilon$;

(d2) if $(1 / 2) \gamma_{1}^{\max } \leq \gamma_{1}^{\max }-\gamma_{3}^{\max }<\gamma_{2}^{\max }, \alpha=\alpha_{1}-\varepsilon$,

where $\alpha_{1}=\left(\gamma_{1}^{\max }-\gamma_{3}^{\max }\right) / \gamma_{1}^{\max }, \alpha_{2}=\gamma_{2}^{\max } / \gamma_{1}^{\max }, \alpha_{3}=\gamma_{2}^{\max } /\left(\gamma_{2}^{\max }+\gamma_{3}^{\max }\right)$ and $\varepsilon$ is small and positive.

Example 1. Discuss the optimal solution for the following initial conditions:

(A) $\rho_{1,0}=0.35, \rho_{2,0}=0.2, \rho_{3,0}=0.9$;

(B) $\rho_{1,0}=0.45, \rho_{2,0}=0.75, \rho_{3,0}=0.15$;

(C) $\rho_{1,0}=0.3, \rho_{2,0}=0.9, \rho_{3,0}=0.8$.

In case $(\mathrm{A})$, we get

$$
r_{1}^{\max }=0.2275, \quad r_{2}^{\max }=0.25, \quad r_{3}^{\max }=0.09,
$$




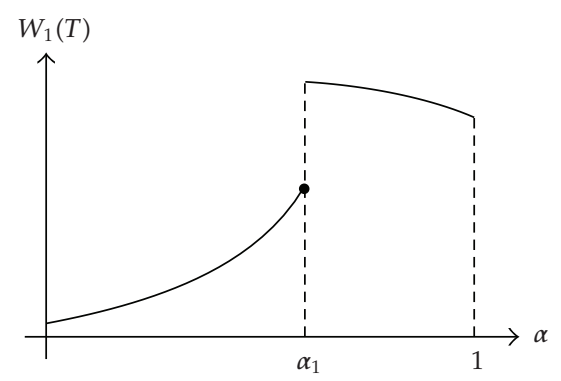

(a)

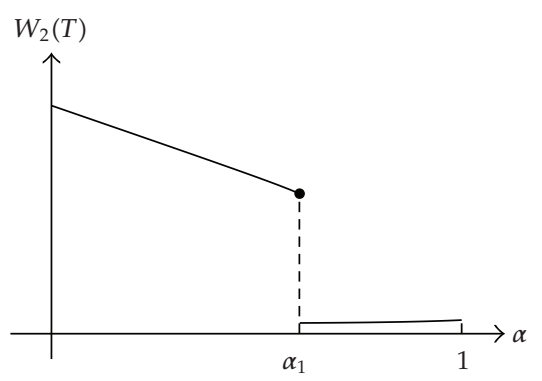

(b)

Figure 2: Case (A): behaviour of $W_{1}$ and $W_{2}$ for sufficiently big.

so condition $\gamma_{3}^{\max }<\gamma_{1}^{\max }<\gamma_{2}^{\max }$ is satisfied. Hence,

$$
\widehat{\gamma}=\left(\widehat{\gamma}_{1}, \alpha \widehat{\gamma}_{1},(1-\alpha) \widehat{\gamma}_{1}\right)
$$

where

$$
\widehat{\gamma}_{1}= \begin{cases}\frac{\gamma_{3}^{\max }}{1-\alpha}, & 0<\alpha \leq \alpha_{1}, \\ \gamma_{1}^{\max }, & \alpha_{1}<\alpha<1,\end{cases}
$$

with $\alpha_{1} \simeq 0.6$. For $T$ sufficiently big, $W_{1}(T)$ and $W_{2}(T)$ have one discontinuity point at $\alpha=\alpha_{1}$, as shown in Figure 2. The optimal control does not exist, but one can choose $\alpha=\alpha_{1}+\varepsilon$.

In case (B), we have that

$$
r_{1}^{\max }=0.2475, \quad r_{2}^{\max }=0.1875, \quad r_{3}^{\max }=0.25,
$$

hence condition $\gamma_{2}^{\max }<\gamma_{1}^{\max }<\gamma_{3}^{\max }$ holds. Then, the solution to the RP at $J$ is

$$
\widehat{\gamma}=\left(\widehat{\gamma}_{1}, \alpha \widehat{\gamma}_{1},(1-\alpha) \widehat{\gamma}_{1}\right)
$$

where

$$
\widehat{\gamma}_{1}= \begin{cases}\gamma_{1}^{\max }, & 0<\alpha \leq \alpha_{2}, \\ \frac{r_{2}^{\max }}{\alpha}, & \alpha_{2}<\alpha<1,\end{cases}
$$

with $\alpha_{2} \simeq 0.7575$. For $T$ sufficiently big, the cost functionals $W_{1}(T)$ and $W_{2}(T)$ have one discontinuity point at $\alpha=\alpha_{2}$, as shown in Figure 3. The optimal control exists, and it is $\alpha=1 / 2$, for which

$$
W_{1}(T)=0.347816, \quad W_{2}(T)=1.1565 .
$$




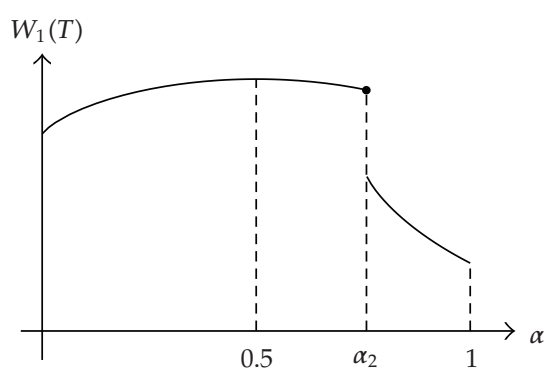

(a)

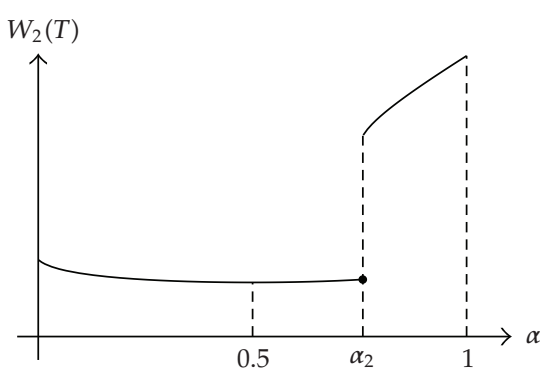

(b)

Figure 3: Case (B): behaviour of $W_{1}$ and $W_{2}$ for $T$ sufficiently big.

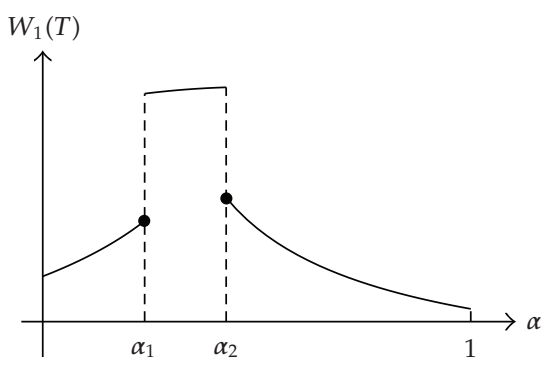

(a)

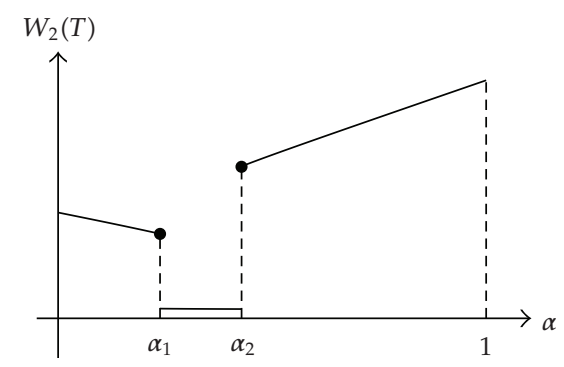

(b)

Figure 4: Case (C): behaviour of $W_{1}$ and $W_{2}$ for $T$ sufficiently big.

In case $(\mathrm{C})$

$$
r_{1}^{\max }=0.21, \quad r_{2}^{\max }=0.09, \quad r_{3}^{\max }=0.16
$$

hence condition $\gamma_{2}^{\max }<\gamma_{3}^{\max }<\gamma_{1}^{\max }$ is satisfied, and we obtain

$$
\widehat{\gamma}=\left(\widehat{\gamma}_{1}, \alpha \widehat{\gamma}_{1},(1-\alpha) \widehat{\gamma}_{1}\right)
$$

where

$$
\widehat{\gamma}_{1}= \begin{cases}\frac{\gamma_{3}^{\max }}{1-\alpha}, & 0<\alpha \leq \alpha_{1}, \\ \gamma_{1}^{\max }, & \alpha_{1}<\alpha<\alpha_{2}, \\ \frac{r_{2}^{\max }}{\alpha}, & \alpha_{2}<\alpha<1,\end{cases}
$$

with $\alpha_{1} \simeq 0.238$ and $\alpha_{2} \simeq 0.428$. The cost functional $W_{1}(T)$ and $W_{2}(T)$, reported in Figure 4 for $T$ sufficiently big, have two discontinuity points at $\alpha=\alpha_{1}$ and $\alpha=\alpha_{2}$. Hence, an optimal value for $\alpha$ does not exist, but we can choose $\alpha=\alpha_{2}-\varepsilon$. 


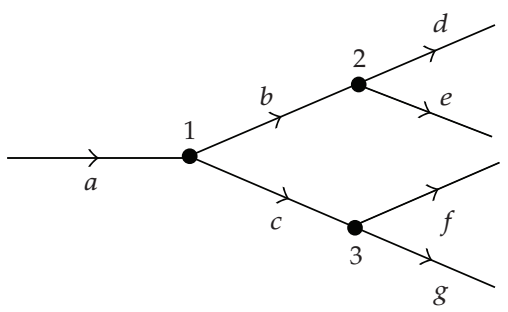

Figure 5: Topology of the symmetric network.

\section{Road Traffic Simulation}

We present some simulation results in order to test the optimization algorithm for the cost functionals. In particular, we analyze the effects of different control procedures, applied locally at each junction, on the global performances of networks and compute the travelling time of a car on assigned paths. For simplicity, from now on we drop the dependence on $T$ from $W_{1}$ and $W_{2}$.

\subsection{A Symmetric Network}

In this subsection, we analyze a symmetric network with three simple junctions of $1 \times 2$ type, labelled by 1,2 , and 3 , see Figure 5 . In particular, the network consists of two inner roads, $b$ and $c$, and five roads, that connect the inner roads to outside: $a, d, e, f$, and $g$. The conservation law with flux function (2.2) is approximated using the Godunov scheme, with space step $\Delta x=0.0125$, and time step, determined by the CFL condition (see [10,11]), equal to 0.5 . We assume initial conditions zero for all roads at the starting instant of simulation $(t=0)$, a 0.3 Dirichlet boundary datum for roads $a, d, e, f$, a 0.9 Dirichlet boundary condition for road $g$ and a time interval of simulation $[0, T]$, where $T=30 \mathrm{~min}$.

Two different choices of the distribution coefficients are considered: (locally) optimal parameters at each junction, given by analytical results (optimal case); random parameters (random case), that is, the distribution coefficients are taken randomly for each road junction when the simulation starts and then are kept constant.

The evolution of $W_{1}$ and of $W_{2}$ are depicted in Figures 6 and 7, reporting with a continuous line the optimal case and with dashed lines various random cases. In some random simulations, the $\alpha$ values are such that a lower traffic density goes to road $g$, with a consequent natural improvement of the network performances. This justifies the fact that some dashed $W_{1}$ and $W_{2}$ curves approach the optimal ones. In other cases, $W_{1}$ rapidly decreases and $W_{2}$ tends to infinity, indicating that the random choice of $\alpha$ provokes congestions on all network roads. However, in any case, the optimal case is better than the others. In fact, it describes the natural situation (that happens on congested real urban networks) in which the traffic is redirected to less congested roads.

\subsection{A Real Urban Network}

This subsection is devoted to the simulation on a portion of the urban network of Salerno, Italy. The network topology, depicted in Figure 8, is characterized by four principal roads. Each of them is divided into segments, labelled by letters: Via Torrione (segments $a, b$, and $c$ ), 


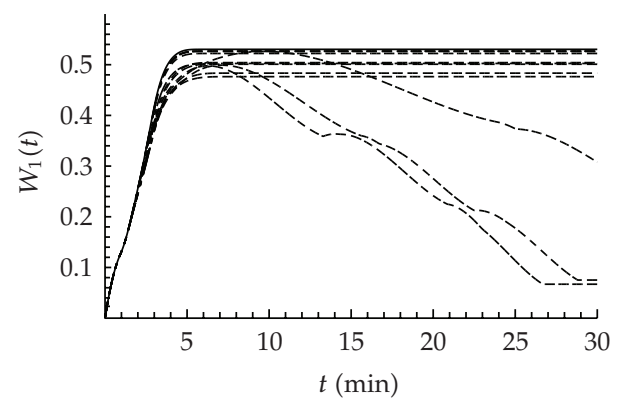

(a)

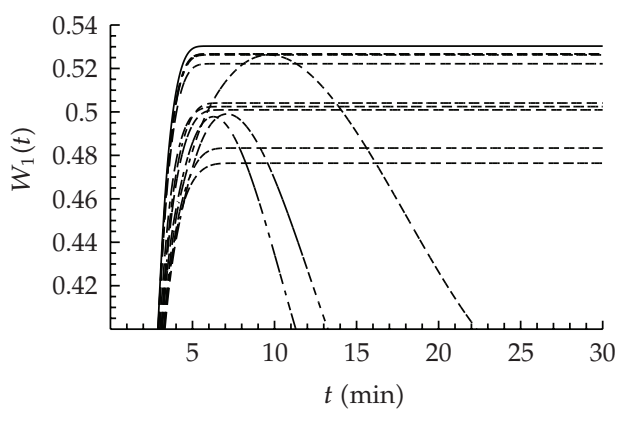

(b)

Figure 6: $W_{1}(t)$ evaluated for optimal distribution coefficients (continuous line) and random choices (dashed lines). (a) evolution in $[0 ; T]$. (b) zoom around the asymptotic optimal values.

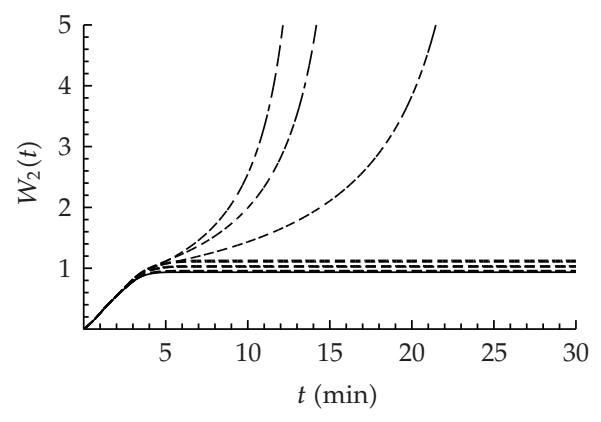

(a)

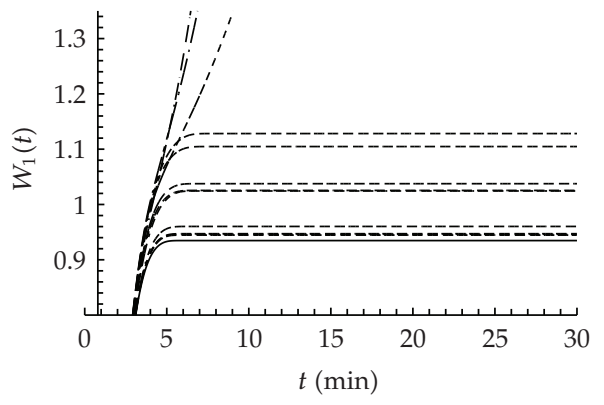

(b)

Figure 7: $W_{2}(t)$ evaluated for optimal distribution coefficients (continuous line) and random choices (dashed lines). (a) evolution in $[0 ; T]$. (b) zoom around the asymptotic optimal values.

Via Leonino Vinciprova (segments $d$ and $e$ ), Via Settimio Mobilio (segments $f, g, h$, and $i$ ), and Via Guercio (segment $l$ ). We distinguish inner roads segments, $b, e, f, g$, and $h$, and external ones, $a, c, d, i, l$. Junctions (indicated by numbers) 1,3 and 5 are of $1 \times 2$ type,while 2 and 4 are of $2 \times 1$ types. The evolution of traffic flows is simulated by the Godunov method with $\Delta x=0.0125, \Delta t=\Delta x / 2$ in a time interval $[0, T]$, with $T=120 \mathrm{~min}$. Initial conditions and boundary data for densities are in Table 1 and have been taken in order to simulate a congestion scenario. Notice that, for junctions 2 and 4, right of way parameters are chosen according to measures on the real network.

In Figure 9, we report the behaviour of $W_{1}$ and $W_{2}$, where optimal simulations are indicated again by continuous lines, while random cases by dashed ones. Random simulations curves of $W_{1}$ are always lower than the optimal ones. In fact, when optimal parameters are used, a flows redistribution occurs on roads, with consequent reduction of congestions at junctions of $1 \times 2$ type. Focus now on $W_{2}$, for which the optimal curve is higher than some random ones. This is not surprising as we deal with the simulation of a high congested asymmetric network. The traffic redirection at congested $1 \times 2$ junctions is of local type and, as expected, benefits occur only on roads and at junctions where the optimization procedure is applied. This is easy deducible considering Via Torrione, which presents a $2 \times 1$ junction, labelled by 2 , where traffic high densities cannot be redirected. 


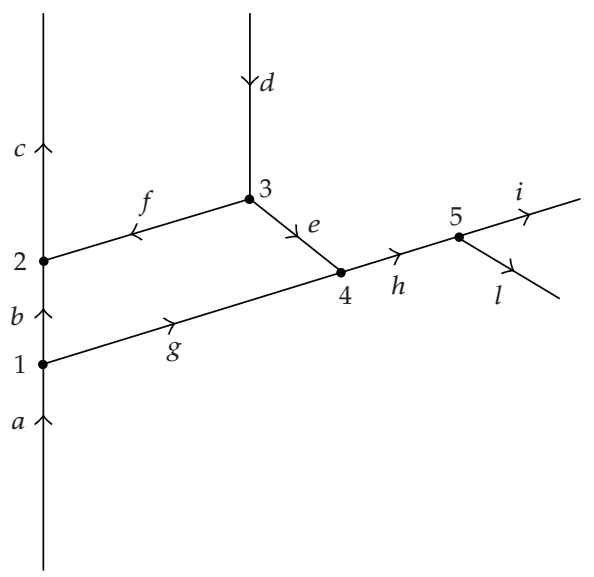

Figure 8: Topology of the portion of the real urban network of Salerno.

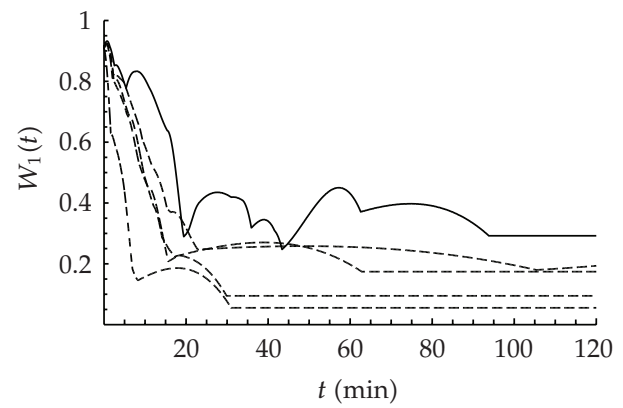

(a)

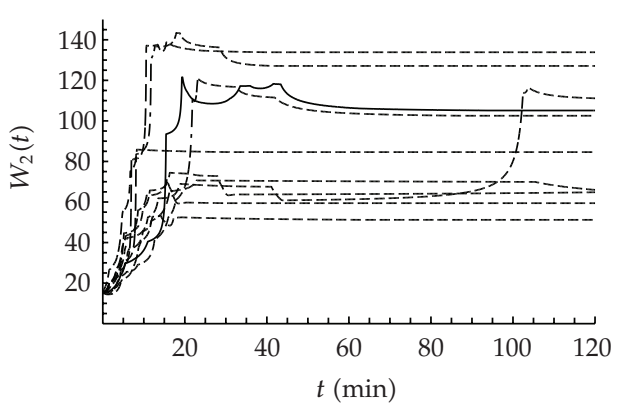

(b)

Figure 9: Evolution in $[0 ; T]$ of $W_{1}(t)$ (a) and $W_{2}(t)$ (b) evaluated for optimal distribution coefficients (continuous line) and random choices (dashed lines).

Table 1: Initial conditions, boundary data and right of way parameters for roads of the network.

\begin{tabular}{lccc}
\hline Road & Initial condition & Boundary data & Right of way parameters \\
\hline$a$ & 0.2 & 0.3 & $/$ \\
$b$ & 0.2 & $/$ & 0.2 \\
$c$ & 0.2 & 0.9 & 0.4 \\
$d$ & 0.2 & 0.3 & $/$ \\
$e$ & 0.8 & $/$ & $/$ \\
$f$ & 0.2 & $/$ & 0.8 \\
$g$ & 0.75 & $/$ & 0.6 \\
$h$ & 0.6 & $/$ & $/$ \\
$i$ & 0.75 & 0.9 & $/$ \\
$l$ & 0.75 & 0.9 & $/$ \\
\hline
\end{tabular}

Even if the right of way parameters which characterize $2 \times 1$ junctions are optimized according to the values in [8], traffic conditions almost remain the same. Hence, although optimal 


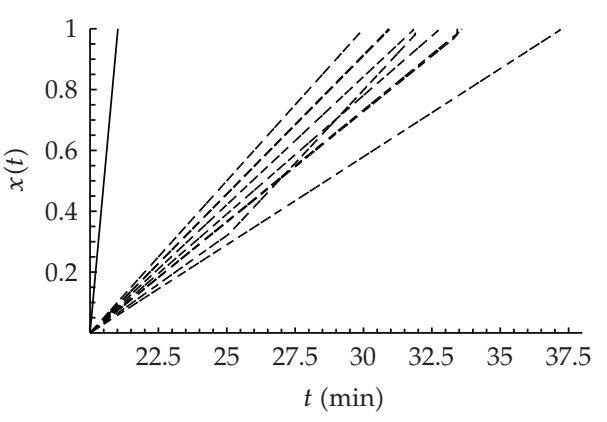

(a)

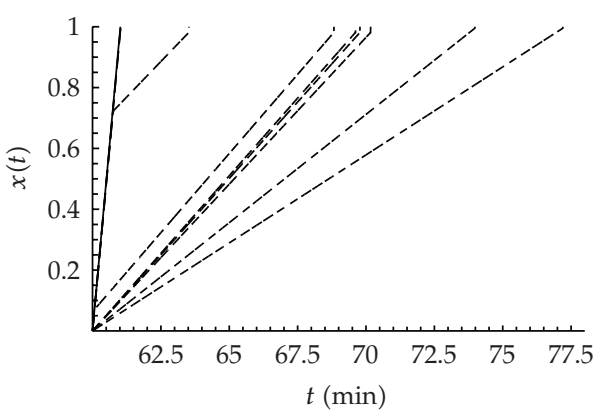

(b)

Figure 10: Evolution of a car trajectory $x(t)$ along road $g$ with initial travel times $t_{0}=20(\mathrm{a})$ and $t_{0}=60$ (b), evaluated for optimal distribution coefficients (continuous lines) and random choices (dashed lines).

distribution coefficients are used, high traffic densities affect some roads and this justifies that the optimal curve is not the lowest for $W_{2}$.

Suppose that a car travels along a path in a network, whose traffic evolution is modeled by (2.1). The position of the driver $x=x(t)$ is obtained solving the Cauchy problem:

$$
\begin{gathered}
\dot{x}=v(\rho(t, x)), \\
x\left(t_{0}\right)=x_{0},
\end{gathered}
$$

where $x_{0}$ is the initial position at the initial time $t_{0}$, while $v=1-\rho$. Using numerical methods, described in [13], we aim to estimate the driver travelling time and to prove the goodness of the optimization results. First, we compute the car trajectory along road $g$ and the time needed for covering it in optimal case and random cases; then, we fix a car path within the Salerno network and study the exit time evolution versus the initial travel time $t_{0}$ (the time in which the car enters into the network).

In Figure 10, we assume that the car starts its own travel at the beginning of road $g$ at the initial times $t_{0}=20$ (a) and $t_{0}=60(\mathrm{~b})$ and compute the trajectories $x(t)$ along road $g$, in optimal case (continuous line) and random cases (dashed lines). Although initial times $t_{0}$ are different, the evolution $x(t)$ in the optimal case has always a higher slope with respect to trajectories in random cases because traffic levels are low. When random choices of parameters $\alpha$ are used, higher boundary conditions for roads $c, i$, and $l$ cause an increase in density values by shocks propagating backwards. Hence, car velocities are reduced, travel times become longer and so exit times from road $g$.

In Table 2, we collect the exit times $T_{\text {out }}$ from road $g$ (the times needed to go out from road $g$ ), for the optimal choice of distribution coefficients (opt) and random choices $\left(r_{i}, i=\right.$ $1, \ldots, 9)$, assuming $t_{0}=20$.

The exit time $T_{\text {out }}(t)$ from road $g$ versus the initial time $t_{0}$, assuming that the car starts its path from the beginning of the road is shown in Figure 11(a). Because of the decongestion effects, the choice of optimal coefficients (continuous lines) allows to obtain an exit time lower than the other cases (dashed lines). Notice that the exit time becomes stable after a certain initial time value $\left(t_{0} \simeq 18.5\right.$ for the optimal distribution choice, unlike the random cases, for which $t_{0} \simeq 35.5$ and $t_{0} \simeq 42$ ). 
Table 2: Initial conditions, boundary data and right of way parameters for roads of the network.

\begin{tabular}{lc}
\hline Simulations & $T_{\text {out }}$ \\
\hline opt & 1.02 \\
$r_{1}$ & 9.955 \\
$r_{2}$ & 10.89 \\
$r_{3}$ & 10.93 \\
$r_{4}$ & 11.82 \\
$r_{5}$ & 11.895 \\
$r_{6}$ & 12.75 \\
$r_{7}$ & 13.43 \\
$r_{8}$ & 13.585 \\
$r_{9}$ & 17.185 \\
\hline
\end{tabular}

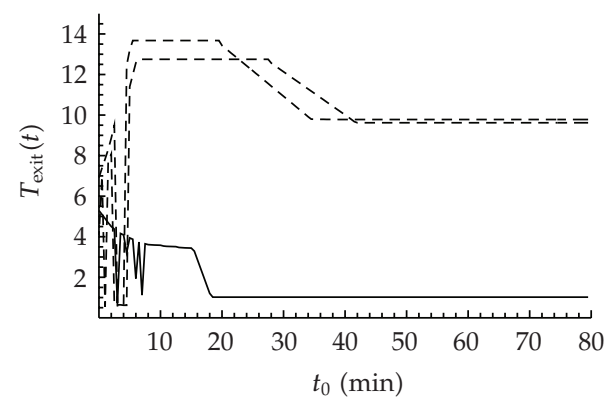

(a)

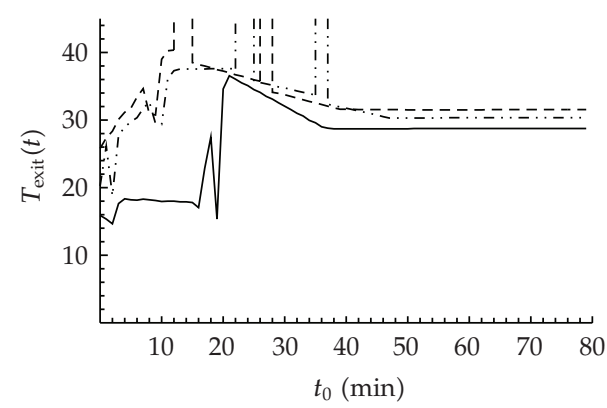

(b)

Figure 11: Exit time from road $g$ versus initial travel time $t_{0}$ in $[0,80]$ (a) and exit time from the path $(a, g, h, l)$ versus initial travel times $t_{0}$ in $[0,80](b)$, evaluated for optimal distribution coefficients (continuous lines) and random choices (dashed and dot dashed lines).

Finally, in Figure 11(b), we consider the exit time $T_{\text {out }}(t)$ from a fixed route, $(a, g, h, l)$, versus the initial times $t_{0}$. Precisely, we study the temporal variation of $T_{\text {out }}$ when a car, starting its trip at the beginning of road $a$ at time $t_{0}$, crosses roads $a, g, h, l$ in order to exit from the network. Also in this case, different choices of distribution coefficients (the optimal one is indicated by a continuous line, unlike the others) affect the time for covering the path. When optimal parameters are not used, $T_{\text {out }}$ tends to infinity at some critical times $t_{c}\left(t_{c} \simeq 13\right.$ and $t_{c} \simeq 27$ for the random case represented by dashed line, and $t_{c} \simeq 23$ and $t_{c} \simeq 36$ for the random case with dot-dashed line). This occurs because the car cannot enter road $g$ or road $h$, since the traffic within them is blocked. For times greater than critical ones, traffic densities become stable (a light decongestion allows the car to reach the destination) and exit times reach a steady value $\left(T_{\text {out }} \simeq 31.5\right.$ and $\left.T_{\text {out }} \simeq 30.35\right)$ at times, respectively, $t_{0} \simeq 42$ and $t_{0} \simeq 46$. On the contrary, when optimal parameters are used, the exit time does not tend to infinity (as the network is never congested) and reaches the steady value $T_{\text {out }} \simeq 28.75$ at time $t_{0} \simeq 38$ (lower, as expected, than steady-state times of simulation with random parameters). 


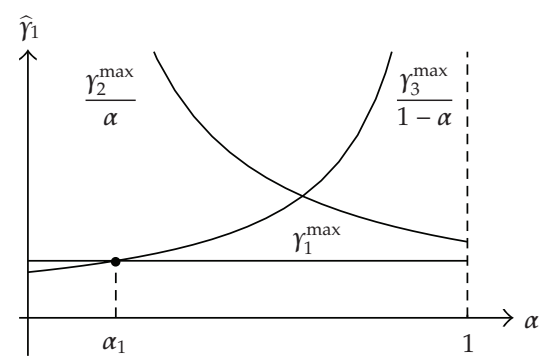

(a)

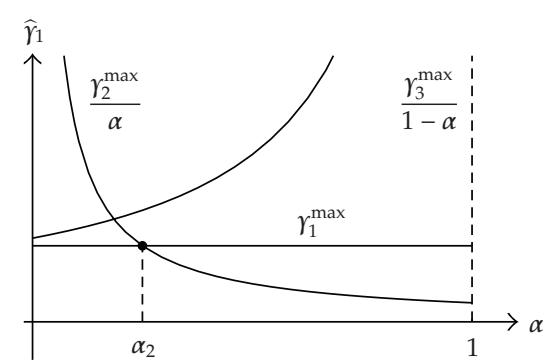

(b)

Figure 12: (a) case $\gamma_{3}^{\max }<\gamma_{1}^{\max }<\gamma_{2}^{\max }$. (b) case $\gamma_{2}^{\max }<\gamma_{1}^{\max }<\gamma_{3}^{\max }$.

\section{Conclusions}

In this paper, an optimization study has been presented to improve the urban traffic conditions in case of special events in which splitting of flows is needed.

The optimization has been made over traffic distribution coefficients at junctions, using two cost functionals, that measure, respectively, the kinetic energy and the average travelling times of drivers, weighted with the number of cars moving on roads. An exact solution has been found for simple junctions having one incoming road and two outgoing roads. The obtained analytical results have been tested through simulations, showing that in some cases a total decongestion effect is possible. This is also confirmed by evaluations of cars trajectories on some roads and fixed routes on the network: using optimal distribution coefficients, times needed to cover paths are the lowest.

\section{Appendix}

We report the proof of Theorem 3.1. Consider new functionals, $\bar{W}_{1}$ and $\bar{W}_{2}$, in which the terms not depending on $\alpha$ are neglected. Since the solution to the RP at $J$ depends on the value of the parameter $\alpha$, we distinguish various cases. Here, for sake of brevity, we analyze some of them.

Assume (see Figure 12(a)) that

$$
r_{3}^{\max }<r_{1}^{\max }<r_{2}^{\max }
$$

then

$$
\widehat{\gamma}_{1}= \begin{cases}\frac{\gamma_{3}^{\max }}{1-\alpha}, & 0<\alpha \leq \alpha_{1}, \\ \gamma_{1}^{\max }, & \alpha_{1}<\alpha<1,\end{cases}
$$


where $\alpha_{1}=\left(\gamma_{1}^{\max }-\gamma_{3}^{\max }\right) / \gamma_{1}^{\max }$. As for $0<\alpha \leq \alpha_{1}, s_{1}=+1, s_{2}=-1$, and for $\alpha_{1}<\alpha<1$, $s_{2}=s_{3}=-1, \bar{W}_{1}$ and $\bar{W}_{2}$ assume the form:

$$
\begin{aligned}
& \bar{W}_{1}= \begin{cases}\frac{1}{1-\alpha}\left(1-\sqrt{1-4 \frac{\gamma_{3}^{\max }}{1-\alpha}}\right)+\frac{\alpha}{1-\alpha}\left(1+\sqrt{1-4 \frac{\alpha}{1-\alpha} \gamma_{3}^{\max }}\right), & 0<\alpha \leq \alpha_{1}, \\
\alpha\left(1+\sqrt{1-4 \alpha \gamma_{1}^{\max }}\right)+(1-\alpha)\left(1+\sqrt{1-4(1-\alpha) \gamma_{1}^{\max }}\right), & \alpha_{1}<\alpha<1,\end{cases}
\end{aligned}
$$

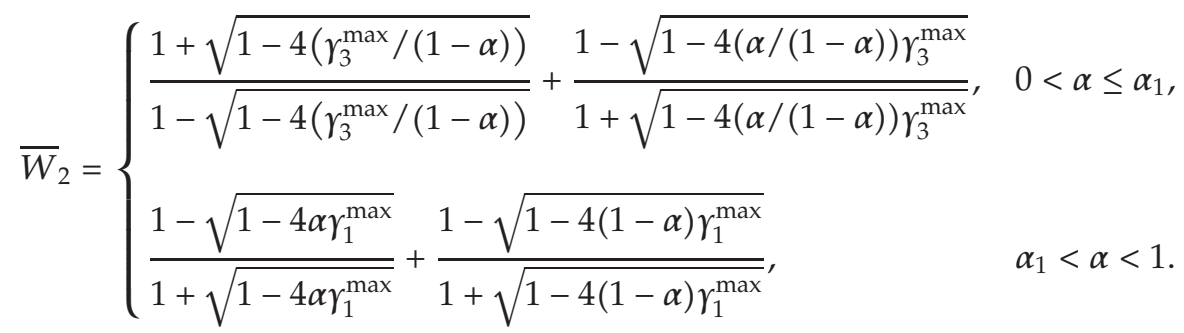

Our aim is to maximize $\bar{W}_{1}$ and to minimize $\bar{W}_{2}$ separately with respect to the parameter $\alpha$.

If $0<\alpha \leq \alpha_{1}$, then

$$
\begin{aligned}
& \frac{\partial \bar{W}_{1}}{\partial \alpha}=2 \frac{\gamma_{3}^{\max }}{(1-\alpha)^{3}}\left[\frac{1}{\mu(1-\alpha)}-\frac{\alpha}{\mu((1-\alpha) / \alpha)}\right]+\frac{1}{(1-\alpha)^{2}}\left[2-\mu(1-\alpha)+\mu\left(\frac{1-\alpha}{\alpha}\right)\right], \\
& \frac{\partial \bar{W}_{2}}{\partial \alpha}=\frac{4 \gamma_{3}^{\max }}{\mu((1-\alpha) / \alpha)[1+\mu((1-\alpha) / \alpha)]^{2}(1-\alpha)^{2}}-\frac{4 \gamma_{3}^{\max }}{\mu(1-\alpha)[\mu(1-\alpha)-1]^{2}(1-\alpha)^{2}}
\end{aligned}
$$

where $\mu(\alpha)=\sqrt{1-4\left(\gamma_{3}^{\max } /(1-\alpha)\right)}$, and hence the functionals $\bar{W}_{1}$ and $\bar{W}_{2}$ are, respectively, an increasing and a decreasing function.

If $\alpha_{1}<\alpha<1$, we get

$$
\begin{aligned}
& \frac{\partial \bar{W}_{1}}{\partial \alpha}=2 \gamma_{1}^{\max }[\eta(1-\alpha)-\eta(\alpha)]+\zeta(\alpha)-\zeta(1-\alpha), \\
& \frac{\partial \bar{W}_{2}}{\partial \alpha}=2 \gamma_{1}^{\max }[\chi(\alpha)-\chi(1-\alpha)+\theta(\alpha)-\theta(1-\alpha)],
\end{aligned}
$$

where

$$
\begin{gathered}
\zeta(\alpha)=\sqrt{1-4 \alpha \gamma_{1}^{\max }}, \quad \eta(\alpha)=\frac{\alpha}{\zeta(\alpha)}, \\
\chi(\alpha)=\frac{1-\zeta(\alpha)}{[1+\zeta(\alpha)]^{2} \zeta(\alpha)}, \quad \theta(\alpha)=\frac{1}{[1+\zeta(\alpha)] \zeta(\alpha)} .
\end{gathered}
$$


Since

$$
\left.\left.\frac{\partial \bar{W}_{1}}{\partial \alpha} \geq 0 \Longleftrightarrow \alpha \in\right] 0, \frac{1}{2}\right], \quad \frac{\partial \bar{W}_{2}}{\partial \alpha} \geq 0 \Longleftrightarrow \alpha \in\left[\frac{1}{2}, 1[\right.
$$

we conclude that $W_{1}$ and $W_{2}$ are maximized and minimized, respectively, for $\alpha=1 / 2$.

Finally, we obtain that

(i) if $\alpha_{1}<1 / 2$, for all $\left.\left.\alpha \in\right] 0, \alpha_{1}[\cup] \alpha_{1}, 1 / 2\right]$,

$$
\frac{\partial \bar{W}_{1}}{\partial \alpha} \geq 0, \quad \frac{\partial \bar{W}_{2}}{\partial \alpha} \leq 0
$$

(ii) if $\alpha_{1} \geq 1 / 2$, for all $\left.\alpha \in\right] 0, \alpha_{1}[$,

$$
\frac{\partial \bar{W}_{1}}{\partial \alpha}>0, \quad \frac{\partial \bar{W}_{2}}{\partial \alpha}<0
$$

Moreover,

$$
\lim _{\alpha \rightarrow \alpha_{1}^{+}}\left[\bar{W}_{1}(\alpha)-\bar{W}_{1}\left(\alpha_{1}\right)\right]>0, \quad \lim _{\alpha \rightarrow \alpha_{1}^{+}}\left[\bar{W}_{2}\left(\alpha_{1}\right)-\bar{W}_{2}(\alpha)\right]>0
$$

Hence, we conclude that

(i) if $\alpha_{1}<1 / 2, W_{1}$ and $W_{2}$ are optimized for $\alpha=1 / 2$;

(ii) if $\alpha_{1} \geq 1 / 2$, the optimal value for both $W_{1}$ and $W_{2}$ does not exist. One can choose $\alpha=\alpha_{1}+\varepsilon$, with $\varepsilon$ small and positive constant.

In the particular case $\gamma_{3}^{\max }<\gamma_{1}^{\max }=\gamma_{2}^{\max }$, the analysis is unchanged. If $\gamma_{1}^{\max }=\gamma_{3}^{\max }<$ $\gamma_{2}^{\max }$ both $W_{1}$ and $W_{2}$ are optimized for $\alpha=1 / 2$.

Assume that (Figure 12(b))

$$
r_{2}^{\max }<\gamma_{1}^{\max }<\gamma_{3}^{\max }
$$

We have

$$
\widehat{\gamma}_{1}= \begin{cases}\gamma_{1}^{\max }, & 0<\alpha \leq \alpha_{2}, \\ \frac{\gamma_{2}^{\max }}{\alpha}, & \alpha_{2}<\alpha<1,\end{cases}
$$


where $\alpha_{2}=\gamma_{2}^{\max } / \gamma_{1}^{\max }$. Then, as for $0<\alpha \leq \alpha_{2}, s_{2}=s_{3}=-1$, and for $\alpha_{2}<\alpha<1, s_{1}=+1$, $s_{3}=-1$, we have to maximize

$$
\bar{W}_{1}= \begin{cases}\alpha \sqrt{1-4 \alpha \gamma_{1}^{\max }}+(1-\alpha) \sqrt{1-4(1-\alpha) \gamma_{1}^{\max },} & 0<\alpha \leq \alpha_{2}, \\ \frac{1}{\alpha}\left(1-\sqrt{1-4 \frac{\gamma_{2}^{\max }}{\alpha}}\right)+\frac{1-\alpha}{\alpha}\left(1+\sqrt{1-4 \frac{1-\alpha}{\alpha} \gamma_{2}^{\max }}\right), & \alpha_{2}<\alpha<1,\end{cases}
$$

and to minimize

$$
\bar{W}_{2}= \begin{cases}\frac{1-\sqrt{1-4 \alpha \gamma_{1}^{\max }}}{1+\sqrt{1-4 \alpha \gamma_{1}^{\max }}}+\frac{1-\sqrt{1-4(1-\alpha) \gamma_{1}^{\max }}}{1+\sqrt{1-4(1-\alpha) \gamma_{1}^{\max }}}, & 0<\alpha \leq \alpha_{2}, \\ \frac{1+\sqrt{1-4\left(\gamma_{2}^{\max } / \alpha\right)}}{1-\sqrt{1-4\left(\gamma_{2}^{\max } / \alpha\right)}}+\frac{1-\sqrt{1-4((1-\alpha) / \alpha) \gamma_{2}^{\max }}}{1+\sqrt{1-4((1-\alpha) / \alpha) \gamma_{2}^{\max }}}, & \alpha_{2}<\alpha<1,\end{cases}
$$

separately with respect to $\alpha$.

Observe that $\bar{W}_{1}$ and $\bar{W}_{2}$ have both a jump at $\alpha=\alpha_{2}$. If $0<\alpha \leq \alpha_{2}, \partial \bar{W}_{1} / \partial \alpha$ and $\partial \bar{W}_{2} / \partial \alpha$ have the same expressions already examined in the previous case. If $\alpha_{2}<\alpha<1$,

$$
\begin{gathered}
\frac{\partial \bar{W}_{1}}{\partial \alpha}=-2 \frac{\gamma_{2}^{\max }}{\alpha^{3}}\left[\frac{1}{\omega(\alpha)}+\frac{1}{\omega(\alpha /(1-\alpha))}\right]+\frac{1}{\alpha^{2}}\left[\omega(\alpha)-\omega\left(\frac{\alpha}{1-\alpha}\right)-2\right] \\
\frac{\partial \bar{W}_{2}}{\partial \alpha}=\frac{4 \gamma_{2}^{\max }}{\alpha^{2}[\omega(\alpha)-1]^{2} \omega(\alpha)}-\frac{4 \gamma_{2}^{\max }}{\alpha^{2}[1+\omega(\alpha /(1-\alpha))]^{2} \omega(\alpha /(1-\alpha))}
\end{gathered}
$$

where $\omega(\alpha)=\sqrt{1-4\left(r_{2}^{\max } / \alpha\right)}$, and it follows that $\bar{W}_{1}$ and $\bar{W}_{2}$ are, respectively, a decreasing and an increasing function. We get

$$
\left.\frac{\partial \bar{W}_{1}}{\partial \alpha} \geq 0, \quad \forall \alpha \in\right] 0, \tilde{\alpha}\left[, \quad \frac{\partial \bar{W}_{2}}{\partial \alpha} \leq 0, \quad \forall \alpha \in\right] 0, \widetilde{\alpha}[
$$

where

$$
\begin{gathered}
\tilde{\alpha}= \begin{cases}\alpha_{2}, & \alpha_{2}<\frac{1}{2}, \\
\frac{1}{2}, & \alpha_{2} \geq \frac{1}{2},\end{cases} \\
\lim _{\alpha \rightarrow \alpha_{2}^{+}}\left[\bar{W}_{1}\left(\alpha_{2}\right)-\bar{W}_{1}(\alpha)\right]>0, \quad \lim _{\alpha \rightarrow \alpha_{2}^{+}}\left[\bar{W}_{2}\left(\alpha_{2}\right)-\bar{W}_{2}(\alpha)\right]<0 .
\end{gathered}
$$


We conclude that

(i) if $\alpha_{2}<1 / 2$, the optimal value for $W_{1}$ and $W_{2}$ is $\alpha=\alpha_{2}$;

(ii) if $\alpha_{2} \geq 1 / 2, W_{1}$ and $W_{2}$ are optimized for $\alpha=1 / 2$.

The obtained optimization results also hold if $\gamma_{2}^{\max }<\gamma_{1}^{\max }=\gamma_{3}^{\max }$; on the contrary, assuming $\gamma_{1}^{\max }=\gamma_{2}^{\max }<\gamma_{3}^{\max }$, the optimal value for both functionals is $\alpha=1 / 2$.

\section{Acknowledgment}

This work is partially supported by MIUR-FIRB Integrated System for Emergency (InSyEme) project under Grant RBIP063BPH.

\section{References}

[1] G. M. Coclite, M. Garavello, and B. Piccoli, "Traffic flow on a road network," SIAM Journal on Mathematical Analysis, vol. 36, no. 6, pp. 1862-1886, 2005.

[2] M. Garavello and B. Piccoli, Traffic Flow on Networks, vol. 1 of AIMS Series on Applied Mathematics, American Institute of Mathematical Sciences (AIMS), Springfield, Mo, USA, 2006.

[3] M. J. Lighthill and G. B. Whitham, "On kinematic waves. II. A theory of traffic flow on long crowded roads," Proceedings of the Royal Society A, vol. 229, pp. 317-345, 1955.

[4] P. I. Richards, "Shock waves on the highway," Operations Research, vol. 4, pp. 42-51, 1956.

[5] A. Bressan, Hyperbolic Systems of Conservation Laws: The One-Dimensional Cauchy Problem, vol. 20 of Oxford Lecture Series in Mathematics and Its Applications, Oxford University Press, Oxford, UK, 2000.

[6] A. Cascone, C. D'Apice, B. Piccoli, and L. Rarità, "Optimization of traffic on road networks," Mathematical Models E Methods in Applied Sciences, vol. 17, no. 10, pp. 1587-1617, 2007.

[7] A. Cascone, C. D'Apice, B. Piccoli, and L. Rarità, "Circulation of car traffic in congested urban areas," Communications in Mathematical Sciences, vol. 6, no. 3, pp. 765-784, 2008.

[8] A. Cutolo, C. D'Apice, and R. Manzo, "Traffic optimization at junctions to improve vehicular flows," Preprint DIIMA, no. 57, 2009.

[9] R. Manzo, B. Piccoli, and L. Rarità, "Optimal distribution of traffic flows at junctions in emergency cases," submitted to European Journal on Applied Mathematics.

[10] E. Godlewski and P.-A. Raviart, Numerical Approximation of Hyperbolic Systems of Conservation Laws, vol. 118 of Applied Mathematical Sciences, Springer, New York, NY, USA, 1996.

[11] S. K. Godunov, "A difference method for numerical calculation of discontinuous solutions of the equations of hydrodynamics," Matematicheskii Sbornik, vol. 47(89), no. 3, pp. 271-306, 1959 (Russian)

[12] J. P. Lebacque, "The Godunov scheme and what it means for first order traffic flow models," in Proceedings of the 13th ISTTT, J. B. Lesort, Ed., pp. 647-677, Pergamon, Oxford, UK, 1996.

[13] G. Bretti and B. Piccoli, "A tracking algorithm for car paths on road networks," SIAM Journal on Applied Dynamical Systems, vol. 7, no. 2, pp. 510-531, 2008. 


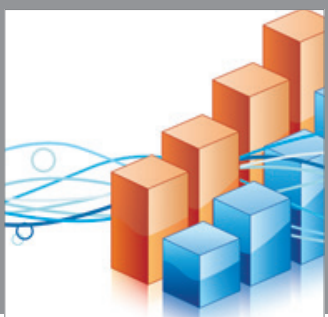

Advances in

Operations Research

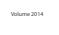

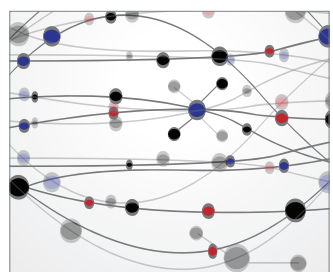

\section{The Scientific} World Journal
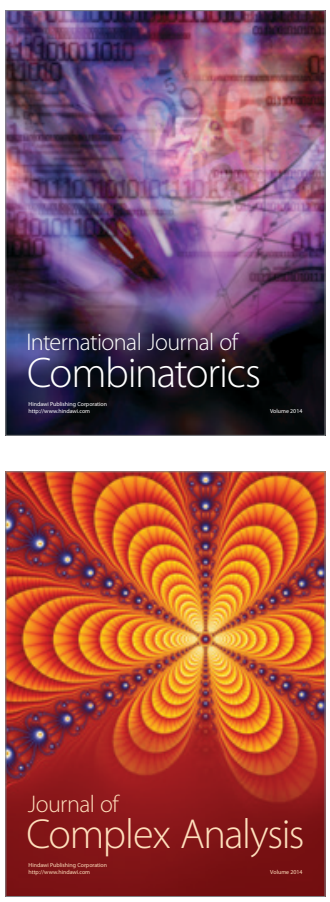

International Journal of

Mathematics and

Mathematical

Sciences
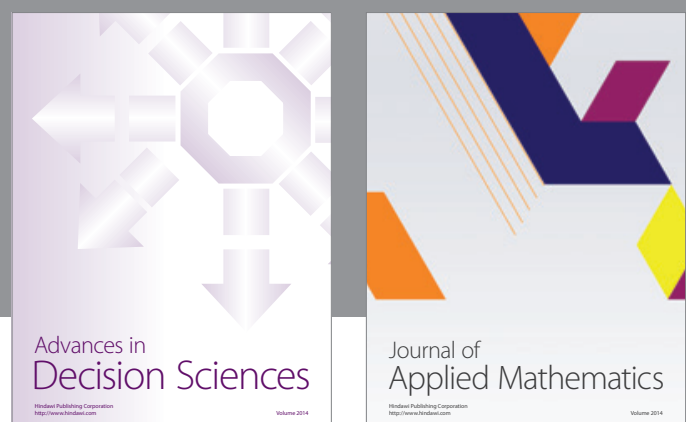

Journal of

Applied Mathematics
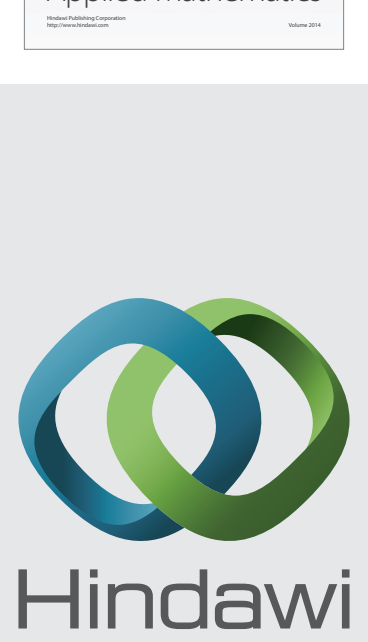

Submit your manuscripts at http://www.hindawi.com
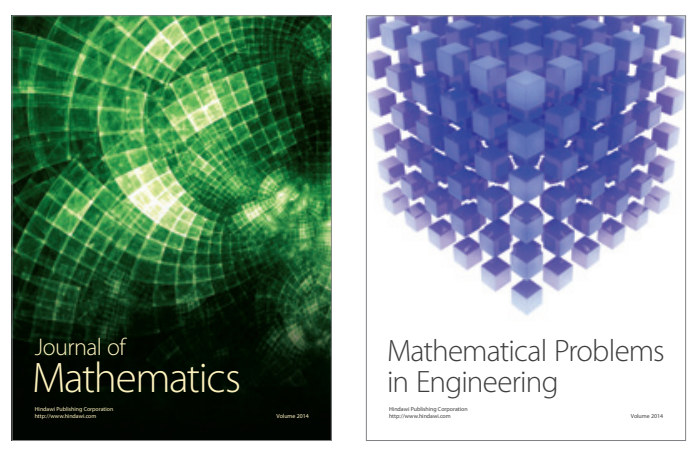

Mathematical Problems in Engineering
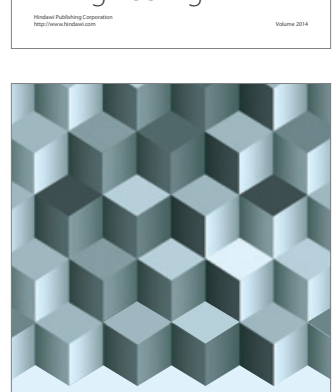

Journal of

Function Spaces
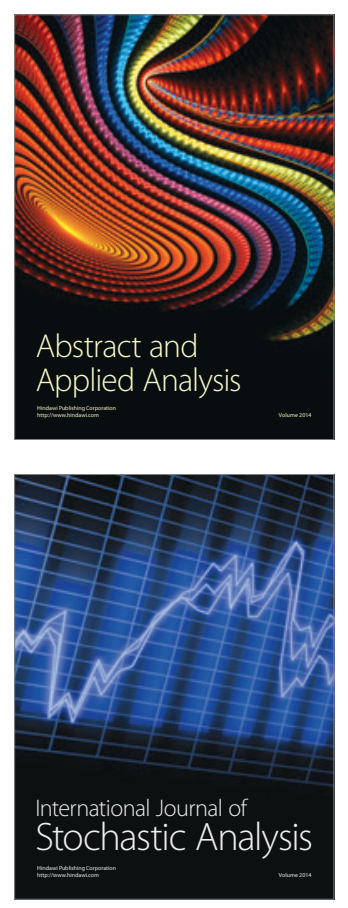

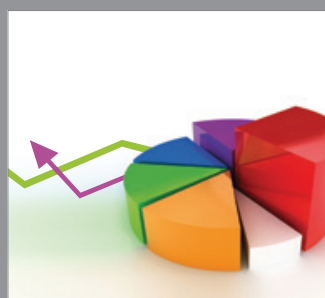

ournal of

Probability and Statistics

Promensencen
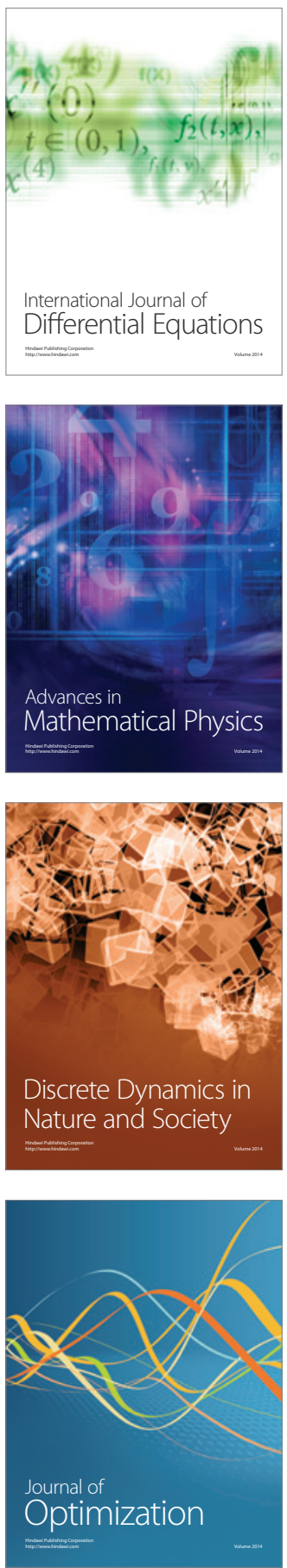